inadequate or limited by side effect. Cannabidiol-CBD, is the major non-psychotropic component of the Cannabis sativa, recent studies on its effectiveness as an anxiolytic, anti-inflammatory, and antipsychotic drug showed promising results, in the setting of chronic pain too.

Objectives: We evaluated the efficacy of CBD drop in pain management in a cohort of SSc patients using standard rating scale VAS, PSQI and HAQ. We further assessed the safety profile and the potential use as opioid-sparing. Methods: From January to November 2019 we consecutively enrolled 31 SSc patients (F/M 26/5, mean age 53.0 $\pm 14.6 \mathrm{SD}$-years) referred to our Scleroderma Unit. All patients satisfied the EULAR/ACR SSc classification criteria. All cases were complicated by painful DUs resistant to analgesics and pain was classified as severe, according to WHO guidelines. CBD drops consist of cannabis sativa seed in olive oil, $10 \% \mathrm{CBD}$, laboratory tested to confirm a tetrahydrocannabinol-THC level $<0.3 \%$. The CBD oil was administrated sublingually twice-a-day. All patients started with CBD 3 drops twice-a-day, and progressively increased to the maximum dosage of 6 drops twice-a-day (from $27.6 \mathrm{~g}$ to $55.2 \mathrm{~g}$ dose/day). All patients continued local/systemic treatments for SSc: $24 / 31$ subjects performed calcium-channel blockers, 31/31 prostanoids infusion, 24/31 anti-endothelin drugs. All subjects were provided with a daily diary to record self-evaluation of pain using VAS, PSQI, hours of sleep per night, use of other analgesics, eventual side effects. HAQ-DI was also administrated. These indicators were assessed baseline and during follow-up. Safety of CBD was evaluated by patient's records of side effects. All data were analyzed by paired t-test. This investigation was a monocentric, prospective study. Ethical approval was obtained from the Competent Ethics Committee (protocol n. 282/15) and all participants gave written consent.

Results: CBD was administered for a mean period of $5.9 \pm 3.2 S D$-months. After the first month, VAS decreased from $94.80 \pm 8.72 S D$ to $54.70 \pm 9.40 S D$ $(p<0.0001)$, PSQI decreased from 9.27 $\pm 2.9 S D$ to $4.47 \pm 1.06 S D(p<0.001)$, total hours of sleep increased from $2.56 \pm 1.28$ SD to $5.67 \pm 0.85 S D(p<0.0001)$. The additional analgesic therapy was necessary in $22 / 31$ patients: $6 / 22$ only paracetamol, 12/22 paracetamol+oxycodone reducing the dosage of oxycodone at the minimum, $2 / 22$ oxycodone $20 \mathrm{mg}$ twice-a-day, 2/22 need fentanyl transdermal patch. After 3 months, VAS further reduced to $40.90 \pm 12.90$, PSQI decreased to $3.1 \pm 1.4 \mathrm{SD}$, the mean total hours of sleep per night was $6.10 \pm 0.79 \mathrm{SD}$ and the HAQ-DI decreased from 2.19 $\pm 0.67 \mathrm{SD}$ (baseline) to $0.79 \pm 0.46 \mathrm{SD}$ at the last patients' evaluation. At the end of the observation, 18/31 patients $(58 \%)$ showed DUs healing. We also interestingly reported improvement of dysphagia and appetite in $70 \%$, and an improvement in constipation related to opiods in $48 \%$. No patients experienced severe side effects in particular no psychoactive aspects. Mild side effects, namely dry mouth was referred by $9 / 31(29 \%)$, mild abdominal pain and changes in appetite by $10 / 31$ (32\%). No interaction with other drugs was observed.

Conclusion: Our study suggests that oral CBD is effective and safe in maintaining analgesia in SSc patients with DUs. Furthermore, CBD could be helpful in opioids tapering and to treat dysphagia, even if these observations need focused investigations. In conclusion, CBD might be a useful tool to manage chronic pain in SSc-DUs. These data provide a compelling rationale for further research to clarify the therapeutic potential of CBD in SSc.

Disclosure of Interests: None declared

DOI: 10.1136/annrheumdis-2021-eular.918

\section{POS0838 1 EPIDEMIOLOGY AND TRENDS IN SURVIVAL OF SYSTEMIC SCLEROSIS IN OLMSTED COUNTY: A POPULATION-BASED STUDY (1980-2018)}

C. Coffey ${ }^{1}$, Y. Radwan ${ }^{2}$, A. Sandhu ${ }^{3}$, C. S. Crowson ${ }^{1,4}$, P. Bauer ${ }^{5}$, E. Matteson ${ }^{1,4}$, A. Makol ${ }^{1}$. ${ }^{1}$ Mayo Clinic, Division of Rheumatology, Rochester, MN, United States of America; ${ }^{2}$ Michigan State University, Internal Medicine, Lansing, MI, United States of America; ${ }^{3}$ Kettering Health, Internal Medicine, Kettering, $\mathrm{OH}$, United States of America; ${ }^{4}$ Mayo Clinic, Health Sciences Research, Rochester, MN, United States of America; ${ }^{5}$ Mayo Clinic, Division of Pulmonary and Critical Care, Rochester, MN, United States of America

Background: Systemic sclerosis (SSc) is a complex immune-mediated disease with heterogeneous manifestations, which is characterized by vasculopathy and fibrosis of the skin and visceral organs. Mortality associated with SSc exceeds that of other rheumatic diseases, though population-based studies assessing recent trends in survival are lacking.

Objectives: We aimed to determine the incidence and prevalence of physician-diagnosed SSc in a population-based cohort over a 39-year time period, and assess for trends in survival over time.

Methods: Medical records of patients with a diagnosis or suspicion of SSc in a geographically well-defined area from Jan 1, 1980 to Dec 31, 2018 were reviewed to identify incident cases of SSc. Cases were defined by physician diagnosis of SSc, and fulfillment of the 2013 ACR/EULAR classification criteria was ascertained. Prevalent cases of SSc on Jan 1, 2015 were also identified. Incidence and prevalence rates were age- and sex-adjusted to the 2010 U.S. white population. Survival rates were compared with expected rates in the general population.

Results: 85 incident cases of SSc (91\% female, mean age $55.4 \pm 16$ y) and 49 prevalent cases on Jan 1, 2015 were identified. Patients had a mean 11.7 (SD 9.4) years of follow-up available. The overall age and sex adjusted annua incidence for $1980-2018$ was 2.5 (95\% Cl: $2.0-3.1)$ per 100,000 population, with no change in incidence over time $(p=0.32)$. The age-adjusted incidence was 4.4 (95\% Cl: 3.4-5.4) for females, and 0.56 (95\% Cl: $0.16-0.96)$ for males per 100,000 population. The age- and sex-adjusted prevalence on Jan 1, 2015 was 43.6 (95\% Cl: 31.3-55.8) per 100,000 population.

77 (91\%) patients fulfilled the 2013 classification criteria; 38 (45\%) fulfilled 1980 criteria. $70(82 \%)$ had limited cutaneous involvement, $12(14 \%)$ had diffuse cutaneous involvement, and $3(4 \%)$ had sine scleroderma. At SSc diagnosis, $80(94 \%)$ patients had Raynaud's, 43 (51\%) had sclerodactyly, 39 (46\%) had telangiectasias, 14/48 (29\%) had abnormal nailfold capillaries, 16/35 (46\%) had digital ulcers or fingertip scarring, 8 (9\%) had interstitial lung disease (ILD), and 7 (8\%) had pulmonary arterial hypertension (PAH). 77/82 patients (91\%) had a positive antinuclear antibody. $44(52 \%)$ had a known SSc-related autoantibody: 32 (73\%) with anti-centromere, 9 (20\%) with anti-Scl-70, and 4 (9\%) with anti-RNA-polymerase III.

Survival was $77 \%(95 \% \mathrm{Cl}: 69-87)$ at 5 years, $66 \%(95 \% \mathrm{Cl}: 56-78)$ at 10 years, and $42 \%(95 \% \mathrm{Cl}: 30-57)$ at 20 years, with no evidence of improved survival over time $(p=0.46)$. Age (Hazard ratio [HR]: 1.49 per 10 year increase; $95 \% \mathrm{Cl}$ 1.19-1.88), smoking at time of diagnosis (HR: $2.37 ; 95 \% \mathrm{Cl}: 1.05-5.34$ ), digital ischemia (HR: 2.54; 95\% Cl: 1.33-4.87), ILD (HR: 4.00; 95\% Cl: 2.11-7.59), and PAH (HR: 4.30; 95\% Cl: 2.24-8.25) had significant associations with mortality Survival of patients with SSc was poorer than the general population (standardized mortality ratio: $2.48 ; 95 \% \mathrm{Cl}: 1.76-3.39)$.

Conclusion: The average incidence of SSc in this population-based cohort spanning 39 years was 2.5 per 100,000 population, with no change in incidence over time. Age, smoking, digital ischemia, ILD and PAH were risk factors fo poorer survival. Overall survival for patients with SSc is worse than that of the general population and shows no improvement over time, suggesting continued need for improved diagnostic and treatment measures.

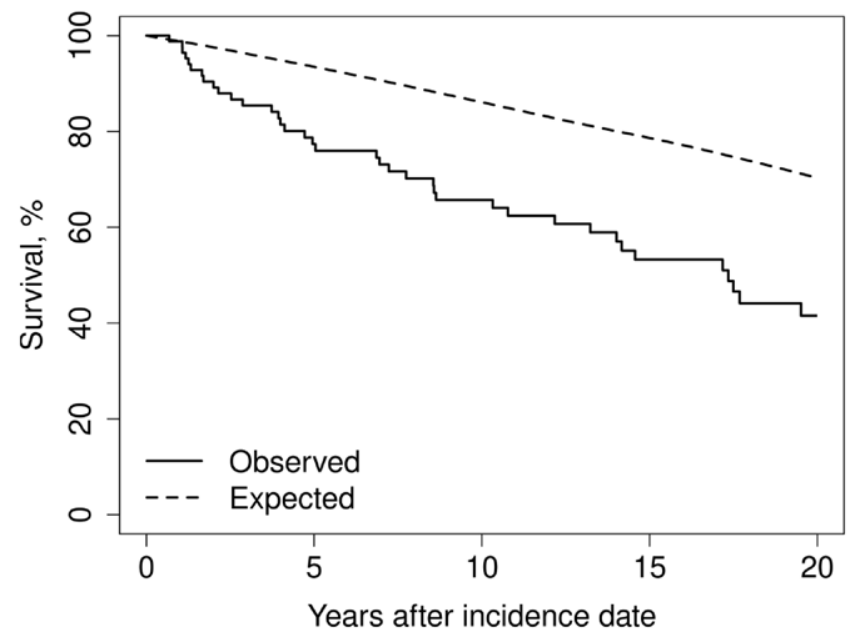

Figure 1. Survival of 85 Olmsted County residents with SSc compared with expected survival rates from Minnesota lifetables (observed: solid line, expected: dashed line).

Disclosure of Interests: None declared

DOI: 10.1136/annrheumdis-2021-eular.919

\section{POS0839 BODY COMPOSITION AND FREQUENCY OF SARCOPENIA IN PATIENTS WITH SYSTEMIC SCLEROSIS}

A. Efremova $^{1}$, O. Nikitinskaya ${ }^{1}$, N. Toroptsova ${ }^{1}$, O. Dobrovolskaya ${ }^{1}$, N. Demin ${ }^{1}$

${ }^{1}$ V.A. Nasonova Research Institute of Rheumatology, Department of Osteoporosis, Moscow, Russian Federation

Background: Sarcopenia can be age associated (primary form) or secondary to chronic disorders, including rheumatic inflammatory disorders. Systemic sclerosis (SSc) is a chronic autoimmune rheumatic disease characterized by widespread vasculopathy, progressive fibrosis of the skin and other internal organs, such as lung, kidneys, gastrointestinal tract, cardiovascular system. Different from the other chronic rheumatic inflammatory disorders, sarcopenia has not been well evaluated in SSc patients. 